\title{
Further developments in the assimilation theory of geometric illusions: The adjacency principle
}

\author{
ALEXANDER W. PRESSEY \\ University of Manitoba, Winnipeg, Manitoba, Canada R3T 2N2 \\ and \\ ROBERT MURRAY \\ Bell Canada, Montreal, Quebec, Canada
}

\begin{abstract}
It is argued that the parallel lines illusion is the basic model for many visual distortions that are produced by geometric patterns. An experiment assessed the effect of moving the contextual contour away from the standard contour in two directions-away from the center of the attentive field and toward the center of the attentive field. The degree of illusion declined as the contextual magnitude moved away from the standard magnitude, but the rate of decline was more rapid when the contextual stimulus was moved away from the center of the attentive field. The results necessitated the addition of a new postulate for the assimilation theory of geometric illusions. This postulate states that the effectiveness of a contextual magnitude decreases as the distance between the contextual magnitude and the standard magnitude increases. The postulate was translated into a mathematical form in a manner analogous to the way" in which the "attentive field" postulate was quantified. The new formula was successful in predicting both the pattern of means and the pattern of variances found in this study. The formula was cross-validated with data from the Ponzo and reversed Mueller-Lyer illusions.
\end{abstract}

In the parallel lines illusion shown in Figure 1, the length of a standard stimulus is altered by the presence of a contextual stimulus. In most cases, the process is one of assimilation in which the standard magnitude becomes more like the contextual magnitude. For example, in Figure 1, the standard magnitude is elongated by the presence of the large contextual magnitude. However, it is likely that under certain conditions contrast (i.e., a phenomenal accentuation of the differences between the standard and contextual magnitudes) would be exhibited.

On the basis of assimilation theory (Pressey, 1967, 1971, 1972), it can be argued that the parallel lines illusion provides the basic paradigm for a large number of geometric illusions, including the MuellerLyer, the Ponzo, the Poggendorff, and the Delboeuf configurations. Thus, for example, the two forms of the Mueller-Lyer illusion reduce to variations of the parallel line illusions, shown in Figure 2, in which $A B$ is equal to $C D$. Unfortunately, very little research has been carried out on this target, despite the fact that systematic variations in the contextual magnitude would give fairly direct evidence on the manner in which contextual stimuli alter the standard stimulus. One major aim of this experiment was to provide empirical data on the effect of the location of a con-

This research was supported by the National Research Council of Canada (AO-177). Reprints may be obtained from the Department of Psychology, University of Manitoba, Winnipeg, Manitoba, Canada R3T 2N2.

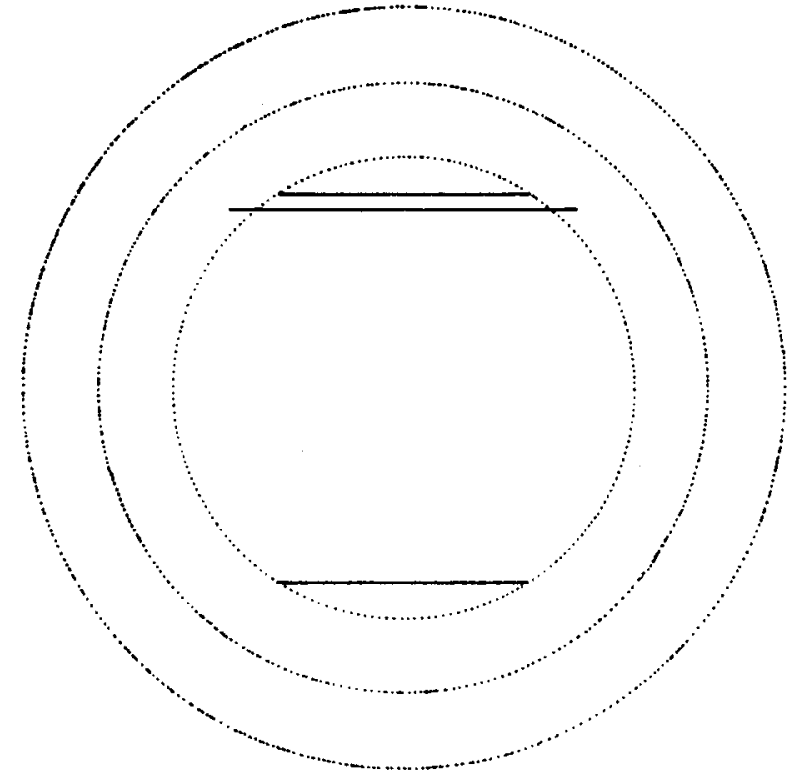

Figure 1. The parallel lines illusion. The short horizontal line in the upper part of the target appears longer than the horizontal line at the bottom of the target. The dotted circles refer to hypothetical attentive fields of different sizes.

textual magnitude on the amount of distortion.

A second aim of this study was to assess the adequacy of assimilation theory to predict the function relating distortion to position of the contextual magnitude. So far, three postulates have been articulated. The first postulate series states that whenever judgments are made of a series of magni- 

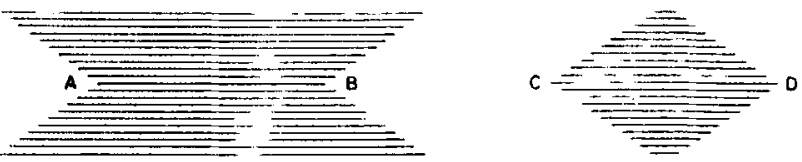

Figure 2. A transformation of the two forms of the MuellerLyer illusion into two forms of the parallel lines illusion.

tudes, the smaller magnitudes in the series will be overestimated and the larger magnitudes will be underestimated. A second postulate states that the degree to which a contextual magnitude is effective depends upon the position of the magnitude within the attentive field. The effectiveness is assumed to decrease as the contextual magnitude moves from the center to the periphery of the field. Finally, a third postulate states that the effectiveness of a contextual magnitude increases as the size difference between the contextual magnitude and the standard magnitude increases. For brevity of expression, the three postulates are called, respectively, the assimilation postulate, the attentive field postulate, and the range postulate.

Recently, a formula has been presented and used to make quantitative predictions of the effects of different stimulus variables on the magnitude of distortion (Pressey \& Bross, 1973; Pressey, Butchard, \& Scrivner, 1971; Pressey \& Sweeney, 1972). The formula is as follows:

$$
I=\frac{1}{N} \sum_{j=1}^{N}\left(1-\frac{D_{c j}}{D_{p}}\right) L_{c j}-L_{s},
$$

where $L_{c j}$ is the length of contextual magnitude $j$; $L_{s}$ is the length of a standard magnitude; $D_{c j}$ is the distance from the center of the attentive field to the most distant point of contextual magnitude $j ; D_{p}$ is the distance from the center of the attentive field to the periphery; and $\mathrm{N}$ is the total number of contextual magnitudes sampled by this estimation procedure, all of which fall entirely within the attentive field.

\section{The Predictions}

The predictions of what should occur in the parallel lines illusion as the position of the contextual magnitude is altered are made with the target shown in Figure 1. The standard magnitude (s) is located in the upper part of the field, and the contextual magnitude (c) is located directly below it. Still further below is the comparison line which is physically equal to $s$. The independent variable is the distance between $\mathrm{c}$ and $\mathrm{s}$ as $\mathrm{c}$ is moved above and below $\mathrm{s}$.

In order to predict the function, the center of the attentive field must be located. An operational definition of an attentive field has been provided (Pressey, 1971). It is the midpoint between the two most extreme elements that are to be judged as set by the instructions. In this case, it would be the midpoint between, say, the left edge of $s$ and the right edge of the comparison magnitude. Figure 1 also shows the periphery of several attentive fields, and these are represented by the dotted circles. The attentive field is presumed to be an organismic variable which differs among individuals. Thus, in the actual predictions, several sizes of attentive fields are sampled in order to estimate the population distribution.

If one uses Formula 1 to predict what will occur as $c$ moves away from $s$, it is clear that divergent trends similar to those shown in Figure $3^{t}$ should be present. Consider first the $c$ which is located above s. As it is displaced upward, it moves further and further away from the center of the field and, according to the attentive field postulate, it should be less and less effective in distorting the standard. However, just the opposite effect should hold when $c$ is displaced downward, because it moves closer and closer to the center of the attentive field. Thus, distortion should increase at least until $c$ reaches the center of the attentive field, at which point the distortion should begin to decrease. Thus, the second objective of this experiment was to determine whether or not the trends depicted in Figure 3 are verified.

\section{EXPERIMENT I}

\section{Method}

Subjects. Seventy-two men and women from introductory psychology courses participated in order to fulfill a course requirement. Only those who had good vision (with or without glasses) were asked to participate.

Materials. Thirty-eight targets, each similar to the target shown in Figure 1, were drawn with black ink on separate $27.8 \times 21.5 \mathrm{~cm}$ sheets of white paper. The standard magnitude was a $50-\mathrm{mm}$ horizontal line that was located $9 \mathrm{~cm}$ below the upper edge of the sheet. The oontextual magnitude was a $70-\mathrm{mm}$ line that was parallel to the standard line. The centers of the two lines fell on the same vertical plane. Each line was about $0.5 \mathrm{~mm}$ wide.

Since the method of production was employed, no comparison line was actually presented. Rather, a small black dot which served as a starting point was drawn in one of two positions. The "left" position was $100 \mathrm{~mm}$ below and $8 \mathrm{~mm}$ to the left of the standard, and the "right" position was $100 \mathrm{~mm}$ below and $8 \mathrm{~mm}$ to the right of the left edge of the standard line.

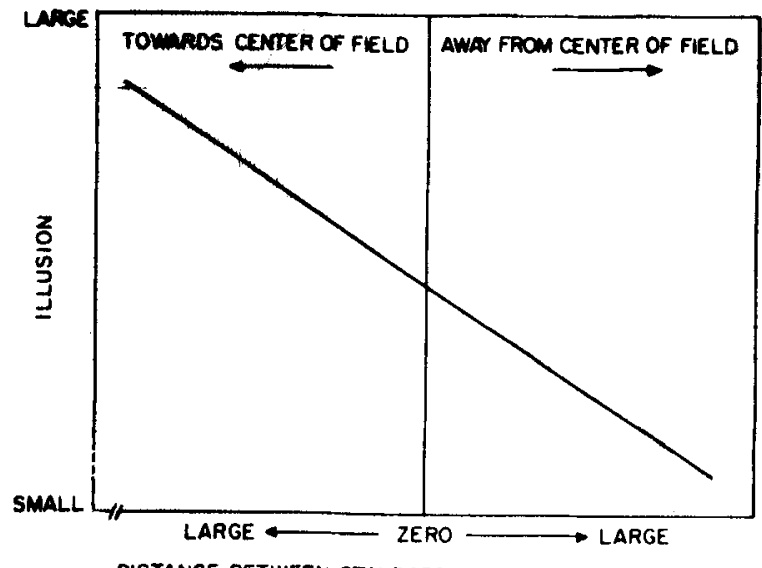

DISTANCE BETWEEN STANDARD AND CONTEXTUAL LINES

Figure 3. Predicted magnitude of illusion in the parallel lines target as a function of the locution of a contextual line. 
The contextual line was located either above or below the standard, and there were nine distances between lines at each location. The distances were $2,4,6,9,12,15,20,25$, and $30 \mathrm{~mm}$. In addition, one control target was employed. Left and right starting positions were available for each target.

The targets were reproduced by offset duplicating procedures. During testing they were placed on a white wooden holder which was $38.5 \mathrm{~cm}$ high and $37 \mathrm{~cm}$ wide. The face of the holder was tilted backward $20^{\circ}$ so that the center of the target would be perpendicular to the line of sight. A chinrest was located directly in front of the holder so that the distance between the subject's eyes and the target was $41 \mathrm{~cm}$.

Procedure. The subject placed his chin in the rest and was shown a sample target. His task was to draw a line which appeared equal to the shorter of the two horizontal lines. (This line was pointed out to him.) He was to start from the dot and draw a horizontal line towards the right of the page. He was asked not to worry too much about making the line exactly straight or exactly horizontal because the experimenter was interested primarily in the apparent length of the line.

The targets were presented manually and were centered on the target holder. The rate of presentation of targets was subjectplaced in the sense that, while the experimenter's rate of presentation was roughly constant, the subjects varied in the time they took to make a response. Each subject made a total of $40 \mathrm{judg}$ ments.

Design. A mixed design was employed in which the position of $c$ (i.e., above or below s) was a between-subjects variable and distance between $s$ and $c$ was a within-subjects variable. Four separate random orders of the 10 targets ( 9 distances and 1 control) were determined for each subject, and the left and right starting positions were counterbalanced within and between subjects. The subjects were assigned to the between-subjects condition alternately as they appeared in the laboratory for testing.

\section{Results and Discussion}

Measurements of the produced line were made with a straight-edge millimeter scale, and these measurements were accurate to within $0.5 \mathrm{~mm}$. For each subject, scores from the four trials were averaged at each distance to yield 10 scores. Then for each subject, the control score was subtracted from the score on the experimental target to yield a measure of illusion. The results are shown in Figure 4. Analysis of variance for a mixed design showed that the variable of Position was significant $(F=6.49$, df $=1,70, p<.01$ ) and the variable of Distance was significant $(F=16.40$, df $=8,560, p<.01)$, but the interaction between Position and Distance was not significant $(F=1.22$, df $=8,560)$. It can be seen from Figure 4 that the amount of illusion was substantially smaller when $c$ was located above $s$ (i.e., at the periphery of the field) than when $c$ was located below s (i.e., at the center of the field). Of course, this trend is consistent with the prediction from the attentive field postulate, which states that the effectiveness of a contextual magnitude decreases from the center to the periphery of the field. At a gross level, then, assimilation theory is substantiated.

However, Figure 4 reveals a major failure for assimilation theory. Formula 1 predicts that, with the particular variables employed in this experiment, the illusion should show a continually increasing function as $\mathrm{c}$ moves away from $\mathrm{s}$ and toward the center of the field. But the fact is that the illusion begins to decrease immediately as the distance between $\mathrm{c}$ and $\mathrm{s}$ increases. Clearly, assimilation theory is in need of elaboration.

The easiest way to handle the discrepancy between the obtained and predicted results is to add another postulate to the existing theory. This postulate would state that the "effectiveness of a contextual magnitude decreases as the distance between the contextual and the focal (standard) magnitude increases."

The idea that the distance between two contours is a critical factor in determining the interaction between these contours has had a long history in perceptual research and theory. This history emerges most clearly when we consider the phenomena of figural aftereffects and the pioneering work of Köhler and Wallach in 1944. Köhler and Wallach discovered what they called the "distance paradox." They found that the distance between the inspection figure and the test figure had a pronounced effect on the degree of distortion that was exhibited. Generally, figural aftereffects were minimal at very short

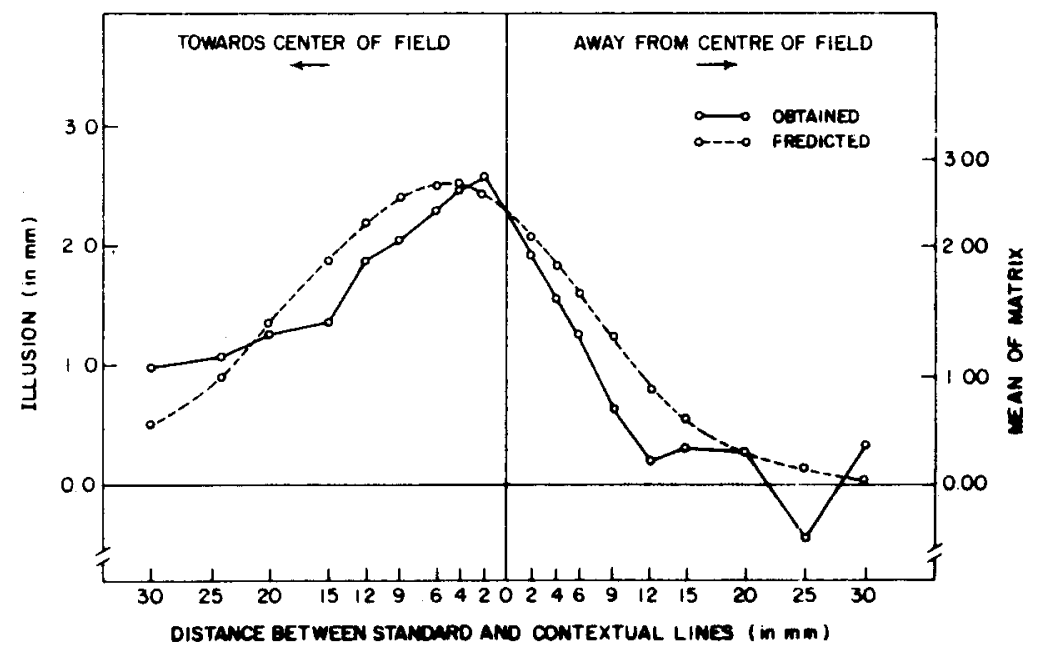

Figure 4. Revised predictions and obtained distortion in the parallel lines target as a function of the location of the contextual contour. 
and very long distances and were maximal at intermediate distances. Köhler and Wallach proposed the concept of "satiation" to explain figural aftereffects, and their explanation involved two prominent features. First, they proposed that satiation extended beyond the contour itself and, second, they argued that the degree of satiation was greatest at the locus of the contour but decreased as the distance from the contour increased. Köhler and Wallach's theory has been criticized on many grounds, but it is interesting that virtually all serious alternatives that have been proposed subsequently (Deutsch, 1964; Ganz, 1966; Osgood \& Heyer, 1952) have implicitly accepted the idea of a distance gradient in which the effect of a contour is maximal at the locus of that contour but decreases as the distance from that contour increases.

Another example of a distance gradient is provided by the neurophysiological evidence from single-cell recordings. In his review of Hubel and Weisel's findings on feature detectors, Coltheart (1971) writes that "although a unit responds best to a contour having the appropriate orientation (i.e., the unit's "preferred orientation"), it will also respond, though less strongly, to contours with somewhat different orientations. The strength of a unit's response is inversely related to the difference between the unit's preferred orientation and the orientation of the stimulus." It should be clear that differences in orientation between two contours can be translated directly into differences in distance between these contours so that, once again, we have evidence that the ability of one contour to affect another contour decreases as the distance between those contours increases.

The notion of a distance gradient has not been as prevalent in theories of geometric illusions, but Restle and Merryman (1969) have shown that the Baldwin illusion is greatly affected by the distance between the standard line and the contextual box. And, although their results are not interpretable unequivocally, one interpretation is that the effect of the box decreased as the distance from the box to the line increased.

Undoubtedly, the most extensive research on the role of spatial separation between contours has been carried out by Gogel and his associates (e.g., Gogel, 1963, 1965, 1970; Gogel \& Mershon, 1969; Mershon $\&$ Gogel, 1970). It is argued that "the effectiveness of cues between points or objects is inversely related to the separation of the points or objects" (Gogel, 1974). Gogel calls this the "adjacency" principle, and it is clear that the application of this principle would go far towards explaining the results shown in Figure 4.

In summary, there seems to be a good deal of evidence to suggest that distance between contours has a profound effect on the interaction of those contours and that the proposed postulate has a substantial empirical base.

However, even if it is accepted that distance between contours is an important variable, there is still the problem of how to incorporate it into the mathematical formula that has been proposed for quantifying predictions. One approach is to conceive of an "interactive field" which is directly analogous to the attentive field in the sense that it would (a) have a center, (b) have a periphery, and (c) be jircular. ${ }^{1}$ Thus, "effectiveness" would again be defined as a ratio, but this time the ratio would be the distance between $c$ and $s$ and the distance from the center to the periphery of this "interactive field." Formula 1 would then be modified to read:

$$
I=\frac{1}{N_{j=1}} \sum_{j}^{N}\left(1-\frac{D_{c j}}{D_{p}}\right)\left(1-\frac{D_{c j}^{\prime}}{D_{p}^{\prime}}\right)\left(L_{c j}-L_{s}\right),
$$

where $D^{\prime}{ }_{c j}$ is the distance from the center of the interactive field to the contextual magnitude $j$, and $D^{\prime} p$ is the distance from the center to the periphery of the interactive field.

An operational definition of a center of an "interactive field" is more difficult to articulate than the center of an attentive field. Suppose are asked to specify in Figure 1 the "distance" between $c$ and s, what would that distance be? Would it be the perpendicular distance, or the distance between the proximal tips of the lines, or the distance between extremities of the two contours? Clearly, one can conceive of an indefinite number of points on each contour and an indefinite (but extremely large) number of combinations of points to yield an indefinite number of distances. But there does not seem to be any one distance which would provide the unique measure of "distance between contours."

As a first approximation (and primarily because of ease in adapting existing computer programs), the distance between the tip of $s$ and the tip of $c$ was taken as a measure of $D^{\prime}{ }_{c j}$. Then, in an attempt to predict the function shown in Figure 4, 12 attentive fields (ranging from 56 to $111 \mathrm{~mm}$, in steps of $5 \mathrm{~mm}$ ) and 11 interactive fields (ranging from 10 to $110 \mathrm{~mm}$ in steps of $10 \mathrm{~mm}$ ) were sampled. All combinations of $D_{p}$ and $D_{p}^{\prime}$ were assessed to yield 132 cells in each matrix, i.e., for each distance between $c$ and s. A predicted function was then graphed for each cell across matrices to yield a family of 132 curves. Visual inspection of these curves showed that certain ranges of values of $D_{p}$ and $D^{\prime} p$ yielded better fits of the obtained data than did others. As a result, a reduced matrix in which $D_{p}$ ranged from 66 to $106 \mathrm{~mm}$ (in steps of $10 \mathrm{~mm}$ ) and $D_{p}^{\prime}$ ranged from 10 to $40 \mathrm{~mm}$ (in steps of $10 \mathrm{~mm}$ ) was employed. The mean of 


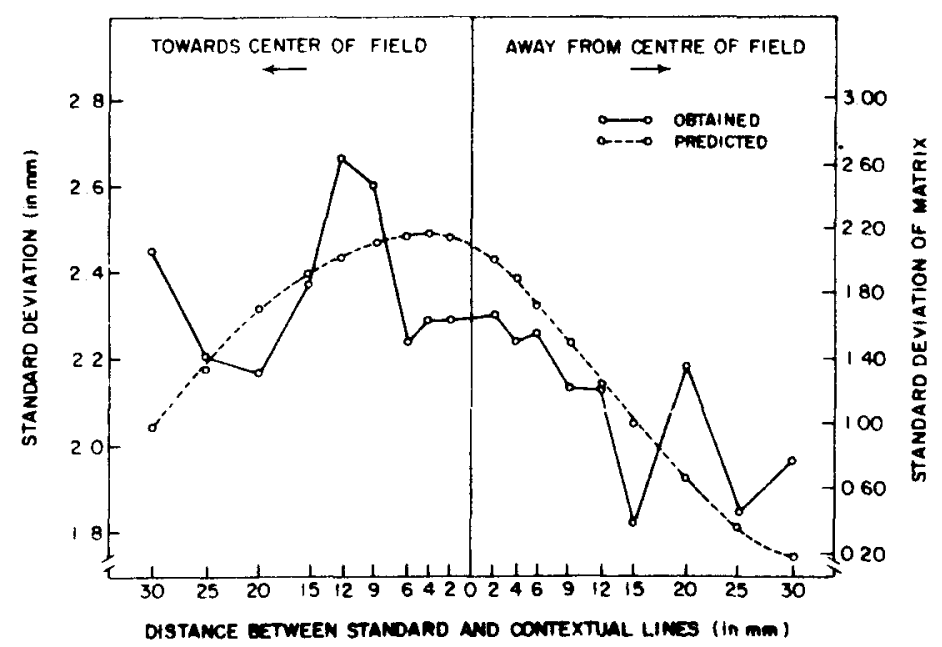

\begin{abstract}
Figure 5. Predicted and obtained standard deviations of scores as a function of the position of the contextual line in the parallel lines target.
\end{abstract}

the 20 values in each reduced matrix was then calculated. The newly predicted means as a function of distance between $c$ and s (both toward and away from the center of the attentive field) are shown in Figure 4 . The fit is obviously very good, and thus the original weakness of assimilation theory has been overcome.

In a previous study (Pressey, et al., 1971), it was noted that selection of several values of attentive field predicted not only means, but also the variability of scores across conditions. It was argued that differences in attentive field may reflect individual differences in the manner in which information is processed. Some individuals process across a restricted area of the display; others deploy attention broadly. Similarly, certain individuals may easily gate out interfering noise when it is close in space to the signal; others may have difficulty in doing so. Thus, it is possible to view each cell in the matrix of predicted scores as corresponding to one type of individual. Moreover, it is reasonable to assert that such individual differences (or perceptual styles) would cause greater variability under stimulus conditions in which a large illusion was expected. Therefore, standard deviations of the 20 values in each reduced matrix (there were 18 such matrices) were calculated, and these were compared with the standard deviations that were obtained in the 18 experimental conditions. The results are shown in Figure 5. Clearly, the relationship between the two functions is more than fortuitous. For example, in both functions, the variability is higher when $c$ is near the center of the field than when $c$ is near the periphery of the field.

Also, both the predicted and obtained functions show a steeper gradient of change when $\mathrm{c}$ is near the periphery of the field than when $c$ is near the center of the attentive field. Thus, Formula 2 provides very promising approximation of both means and variances as the distance between a standard and a contextual line varies in the parallel lines illusion.

Cross-validating the formula. Although the revised formula for predicting perceptual distortion seems powerful enough, we wished to determine whether it would apply to illusions other than the parallel lines illusion. Fortunately, a good deal of stable data are available on both the reversed Mueller-Lyer illusion (Pressey \& Bross, 1973) and the Ponzo illusion (Pressey, 1974). In all cases, the method of production was used in which the distance between the standard line and the comparison line was $100 \mathrm{~mm}$. Also, in every case, the viewing distance was $41 \mathrm{~cm}$ and the length of the standard line was $50 \mathrm{~mm}$. The question we posed was: If exactly the same values of $D_{p}$ and $D^{\prime} p$ were selected as were used in the parallel lines illusion, how well would changes in the reversed Mueller-Lyer illusion be predicted? The reversed Mueller-Lyer illusion is shown in Figure 6. If the gap between the shaft and the apex of the angle is increased, the shrinkage of the shaft will decrease and eventually change into an elongation effect. The pattern of change will vary with changes in the stimulus configuration. A typical function is shown in Figure 7A, in which the illusion is plotted as a joint function of gap and length of oblique lines.

The only complicating factor in using the reversed

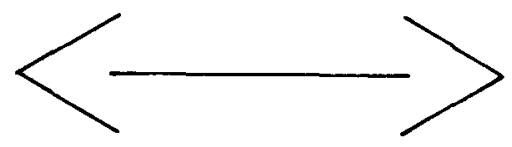

Figure 6. The reversed ingoing form of the Mueller-Lyer illusion. The horizontal line in the upper position appears longer than the lower horizontal line. 


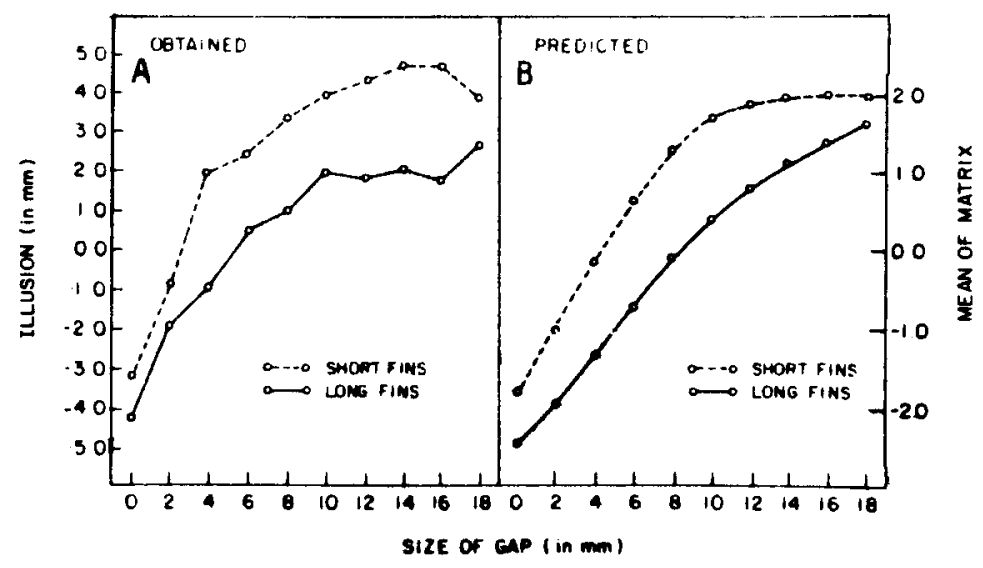

Figure 7. Predicted and obtained means in the Mueller-Lyer illusion as a joint function of gap and length of fin. (The obtained data are from Pressey \& Bross, 1973.)
Mueller-Lyer illusion is that, according to assimilation theory, there are an indefinite number of contextual magnitudes rather than one as in the parallel lines illusion. Therefore, the reversed Mueller-Lyer illusion was converted into forms of the parallel lines illusion by sampling points every $0.1 \mathrm{~mm}$ along the oblique lines and by joining the contralateral points. This gave a series of lines that were parallel to the standard line. The lengths of these lines were determined by the position and length of the oblique fins. This procedure is, of course, identical to the one used by Pressey et al., to make quantitative estimates of the Ponzo illusion.

Formula 2 was applied to the reversed Mueller-Lyer illusions used by Pressey and Bross (1973). $D_{p}$ varied from 66 to $106 \mathrm{~mm}$ in steps of $10 \mathrm{~mm}$, and $D_{p}^{\prime}$ varied from 10 to $40 \mathrm{~mm}$ in steps of $10 \mathrm{~mm}$. All the remaining parameters were obtained empirically from the actual targets that were used in the study. The predicted patterns of means are shown in Figure 7B. Once again, the major features of the empirical functions are predicted. For example, in both obtained and predicted functions, the shrinkage illusion changes to one of elongation as the distance between the shaft and apex of the fin increases. Also in both the obtained and predicted patterns, the size of gap that is necessary to produce a reversed illusion is greater for the long fins than for the short fins.

Figure 8 shows that variability of response is also related to the size of the gap. The obtained function is a U-type, with minimum variability at a gap of $6 \mathrm{~mm}$. The predicted function is also $U$ with a minimum at a gap of $6 \mathrm{~mm}$ (both the predicted and obtained curves in Figure 8 are based on data that were collapsed across fin length in order to provide more stable patterns).

Finally, a great deal of information was available on the manner in which the Ponzo illusion varies as a function of angle of oblique lines (Pressey, 1974). The predicted functions for the values of $D_{p}$ and $D^{\prime} p$ used here yielded the functions illustrated in Fig- ures 9 and 10. It is clear that, once again, the pattern of means and the pattern of variances was predicted exceedingly well by Formula 2.

\section{EXPERIMENT II}

The major purpose of this study was to subject assimilation theory, and especially Formula 2, to a severe test. We sought to determine whether Formula 2 could predict results from an experiment which was designed solely to test the formula. In addition, we wished to utilize a complex design in which several variables and several values of each variable were employed. And finally, we wished to select, as one of our factors, a variable that had not been investigated before. The major constraint, of course, was that the same parameters (viz, size of attentive and interactive fields) as used in the previous predictions would be feasible.

\section{Method}

The Ponzo illusion was chosen as the basic configuration. The new variable that was to be manipulated was the gap between the tips of the standard line and the nearest point of

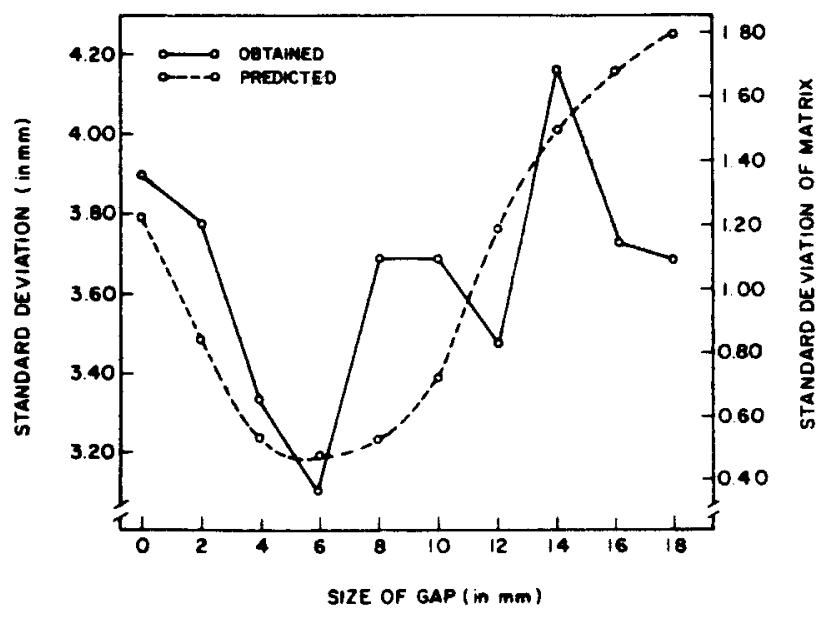

Figure 8. Predicted and obtained variances as a function of gap in the reversed Mueller-Lyer illusion. 


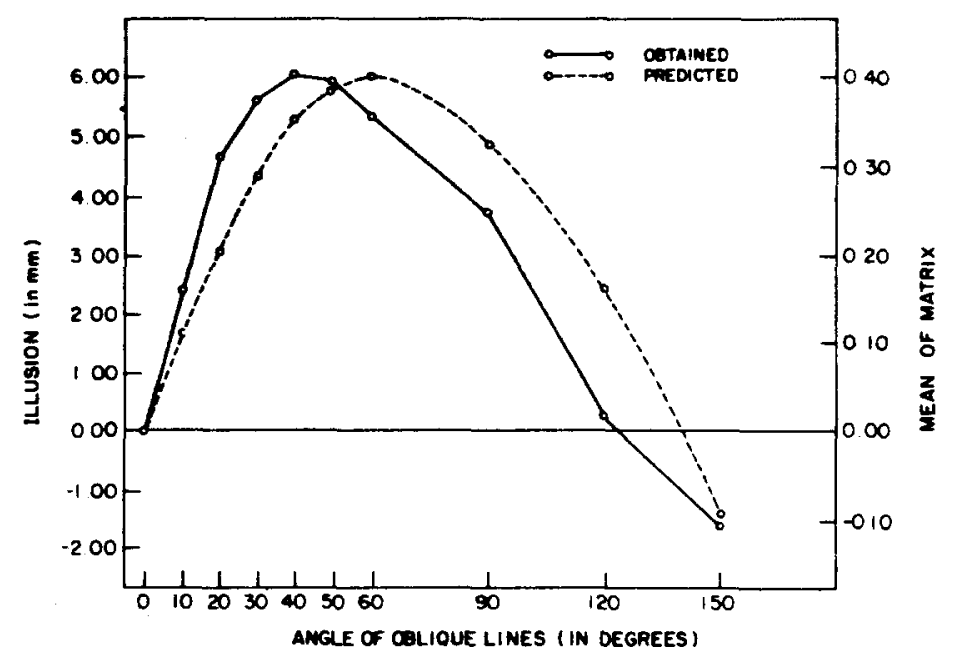

Figure 9. Predicted and obtained means in the Ponzo illusion as a function of angle of oblique lines.

the oblique lines, and the second variable was angle formed by the oblique lines.

Subjects. One hundred and twenty men and women from introductory psychology courses participated in order to fulfill a course requirement. Only those who had good vision (with or without glasses) were asked to participate.

Design. A 4 by 10 mixed design was employed in which there were 4 angles of oblique arms $\left(15^{\circ}, 45^{\circ}, 75^{\circ}\right.$, and $\left.150^{\circ}\right)$ and 10 sizes of gap ranging from 0 to $18 \mathrm{~mm}$ in steps of $2 \mathrm{~mm}$. The variable of Angle was between-subjects, and Gap Size was within-subjects. The subjects were assigned to conditions in order of their appearance in the laboratory and, for each subject, a random order of presenting size of gap was determined. Two starting positions were also employed, and these were given in sucession for each gap. However, order of starting position was counterbalanced between subjects in each group. An equal number of subjects served in each group.

Materials. Forty targets, similar to the one shown in Figure 11, were drawn with black ink on separate $27.8 \times 21.5 \mathrm{~cm}$ sheets of white paper. The standard magnitude was a $50-\mathrm{mm}$ horizontal line. The oblique lines were $100 \mathrm{~mm}$ long and were positioned in such a fashion that $25 \mathrm{~mm}$ of that line was above and $75 \mathrm{~mm}$ was below the standard line. Each line was about $0.5 \mathrm{~mm}$ wide.

Since the method of production was employed, no comparison line was actually presented. Rather, a small black dot, which served as a starting point, was drawn in one of two positions. The "left" position was $100 \mathrm{~mm}$ below and $8 \mathrm{~mm}$ to the left of the standard line, and the "right" position was $100 \mathrm{~mm}$ below and $8 \mathrm{~mm}$ to the right of the left edge of the standard line. The diameter of each dot was about $1 \mathrm{~mm}$.

The target holder, viewing distance, and testing procedure were identical to those used in Experiment $I$.

\section{Results}

Formula 2 was employed to predict the Ponzo illusion as a joint function of angle and gap. All stimulus values were derived from the targets that were used in the experiment, and the parameters of attentive field and interactive field were identical to the ones described earlier. The predictions are shown in Figure 12. Also shown in Figure 12 are the empirical functions that were found in this experiment. The illusion scores were obtained by measuring each produced line by a straight-edge millimeter scale. For each subject, the mean of the two starting positions was obtained and the objective length of the standard line was subtracted from the score. [A control condition was deliberately omitted, since the design already demanded a large number of judgments. Moreover, previous findings (Pressey, 1974) had shown that, with a large number of subjects, the control length was almost identical to the objective length of the standard.]

On the whole, the fit between the predicted and obtained functions is impressive. For example, the pattern of illusion as a function of gap reverses at the $150^{\circ}$ angle and a reversal is nicely shown in the predicted function. What is not well predicted is the main effect of gap. In addition, analysis of the variability of scores showed little correspondence with predicted variability. This was due primarily to the fact that variability did not differ across conditions. However, this lack of congruence between obtained and predicted functions could well be due to the design in which gap was a within-subjects variable. It may be that the relatively flat functions are due to carryover effects from the large number of judgments that each subject had to make. In such a case, the error in prediction would be due to experimental

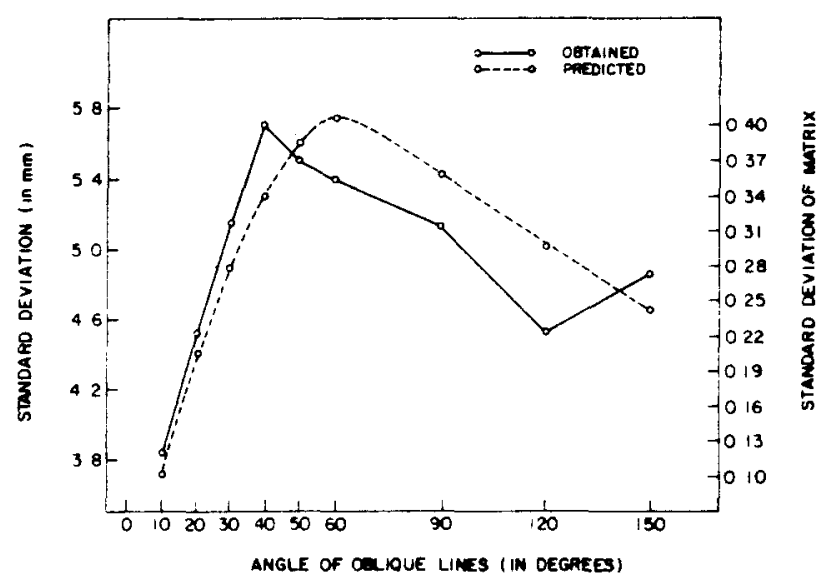

Figure 10. Predicted and obtained and standard deviations in the Ponzo illusion as a function of angle of oblique lines. 


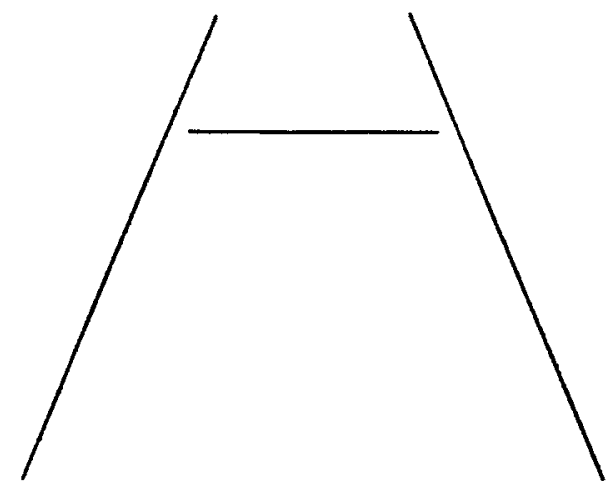

Figure 11. Sample of Ponzo targets employed in this experiment. The dot provides the starting position for drawing the line.

design, and not to the theory.

The current series of studies is, by far, the most stringent test of assimilation theory that has been employed to date. It must be remembered that in all four cases where predictions were made, the same values for the attentive field and the interactive field were employed. Thus, it is difficult to believe that the correspondence between obtained and predicted functions shown here is fortuitous. The more reasonable interpretation is that assimilation theory has specified, fairly well, the manner in which information is processed when the target consists of a geometric illusion.

It is probably appropriate, at this point, to consider the direction that the future developments in assimilation theory should take. Certainly one can continue to specify the variables that affect illusions and integrate these into the theory, as was done in this study. However, continuing such a strategy is questionable because there are more basic problems that must be faced. One of those problems concerns the phenomenon of contrast.

It was recognized long ago (Pressey, 1967) that assimilation is only one of, perhaps, several ways that the organism processes information. Contrast is certainly another way, as is obvious in the Titchener circles and in certain forms of the Delboeuf (Keats, 1964) and Mueller-Lyer illusions (e.g., Fraisse, 1971). But, in terms of theory building, it is extremely difficult to integrate assimilation and contrast into one theory because of the ease with which one can slip into ad hoc explanations. Consider the Belboeuf illusion. When the contextual circle is of a certain size, assimilation occurs; but when it is made much larger, contrast occurs. But what does "much larger" mean and how can one specify it on an a priori basis? We need some rule to tell us when assimilation will occur and when contrast will occur. But such a rule has not been articulated in the past, nor is it easy to articulate it now.

In the development of assimilation theory, the phenomenon of a contrast was assiduously avoided because it seemed necessary first to establish that a cognitive, or information processing, approach was feasible. The present study, along with others, has confirmed that feasibility and the task of integrating contrast into the general framework of assimilation theory should now be given high priority.

\section{REFERENCES}

Coltheart, M. Visual feature-analyzers and aftereffects of tilt and curvature. Psychological Review, 1971, 78, 114-121.

Deutsch, J. A. Neurophysiological contrast phenomena and figural after-effects. Psychological Review, 1964, 17, 19-26.

Fraisse, P. L'integration temporelle des elements des illusions optico-geometriques et l'inversion de l'illusion de Müller-Lyer. L'année Psychologique, 1971, 71, 53-72.

Ganz, L. Mechanisms of the figural after-effect. Psychological Review, 1966, 73, 128-150.

Gogel, W. C. The visual perception of size and distance. Vision Research. 1963, 3, 101-120.

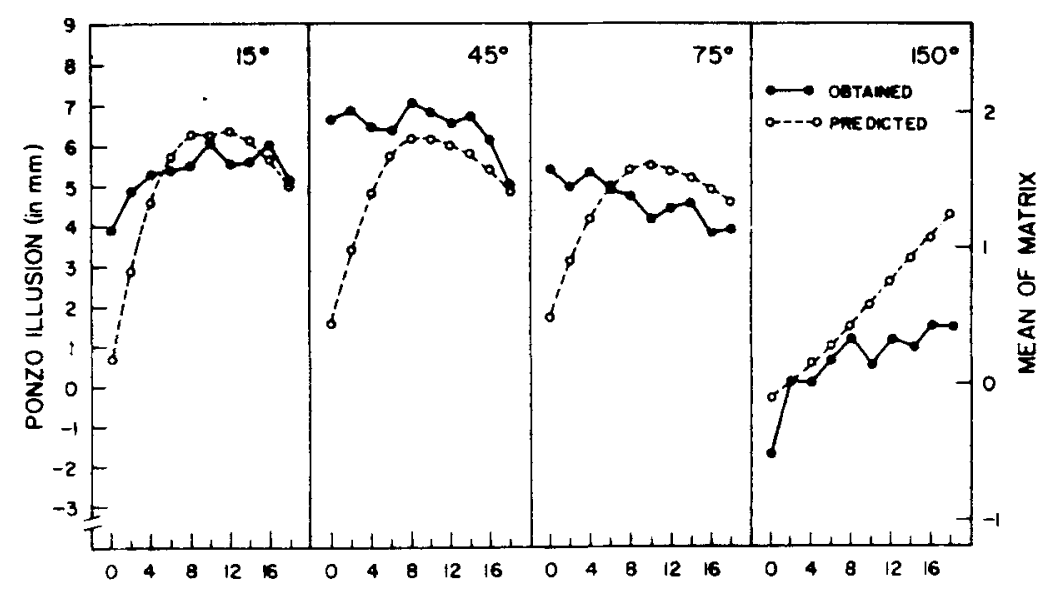

GAP BETWEEN STANDARO AND OBLIQUE LINES (in $\mathrm{mm}$ )
Figure 12. Predicted and obtained functions relating Ponzo illusion to gap and angle. 
Gogel, W. C. Size cues and the adjacency principle. Journal of Experimental Psychology, 1965, 70, 289-293.

GoGEL, W. C. The adjacency principle and three-dimensional visual illusions. Psychonomic Monograph Supplement, $1970,3,153-219$.

GoGEL, W. C. Relative motion and the adjacency principle. Quarterly Joumal of Experimental Psychology, 1974, 26, 425-437.

Gogel, W. C., \& Mershon, D. H. Depth adjacency in simultaneous contrast. Perception \& Psychophysics, 1969, 5, 13-17.

Keats, J. A. An application of the method of paired comparisons to the study of the Delbouf illusion. Australian Journal of Psychology, 1964, 16, 169-174.

KöHLER, W., \& WALLAcr, H. Figural after-effects: An investigation of visual processes. Proceedings of the American Philosophical Society, 1944, 88, 269-357.

MershoN, D. H., \& GoGel, W. C. Effect of stereoscopic cues on perceived whiteness. American Journal of Psychology, $1970,83,55-67$.

Osgood, C. E., \& HeYer, A. W. A new interpretation of figural aftereffects. Psychological Review, 1952, 59, 98-118.

Pressey, A. W. A theory of the Müller-Lyer illusion. Perceptual and Motor Skills, 1967, 25, 569-572.

Pressey, A. W. An extension of assimilation theory to illusions of size, area, and direction. Perception \& Psychophysics, $1971,9,172-176$.

Pressey, A. W. The assimilation theory of geometric illusions: An additional postulate. Perception \& Psychophysics, 1972, 11, 28-30.

Pressey, A. W. Measuring the Ponzo illusion with the method of production. Behavior Research Methods and
Instrumentation, 1974, 6, 424-426.

Pressey, A. W., \& Bross, M. Assimilation theory and the reversed Muller-Lyer illusion. Perception, 1973, 2, 211-217.

Pressey, A. W., Butchard, N., \& Scrivere, L. Assimilation theory and the Ponzo illusion: Quantitative predictions. Canadian Joumal of Psychology, 1971, 25, 486-497.

Pressey, A. W., \& Sweeney, O. Some puzzling results on the Poggendorff illusion. Perception \& Psychophysics, $1972,12,433-437$.

Restie, F., \& Merryman, C. T. Distance and an illusion of length of line. Joumal of Experimental Psychology, 1969, 81, 297-302.

\section{NOTES}

1. There is no necessity for the attentive field or the interactive field to be exactly circular. Indeed, the fact that we have two eyes which are located in a horizontal plane and the fact that space has long been considered to be "anesotropic" would argue against the attentive field or interactive field being exactly circular. Nevertheless, a conception of a circular field does provide a good approximation of what is meant by "decreasing effectiveness across space," and, of course, it is the simplest method of quantifying the theory.

2. We wish to express our thanks to Michael Gunther for testing the subjects in this experiment.

(Received for publication December 3, 1975 revision received March $30,1976$. 